\title{
Intervenção fisioterapêutica nas sequelas de drenagem linfática manual iatrogênica: relato de caso
}

\author{
Physical therapy in sequelae of iatrogenic manual lymphatic drainage: a case report
}

Rogério Eduardo Tacani ${ }^{1}$, Pascale Mutti Tacani ${ }^{2}$, Richard Eloin Liebano ${ }^{3}$

Estudo desenvolvido na UNICID - São Paulo (SP), Brasil.

\section{${ }^{1}$ Fisioterapeuta; Doutorando em} Engenharia Biomédica pela Universidade de Mogi das Cruzes (UMC); Mestre em Ciências do Movimento pela Universidade Guarulhos (UnG); Docente dos cursos de graduação em fisioterapia do Centro Universitário São Camilo e da UnG; Coordenador do Curso de PósGraduação Lato Sensu em Fisioterapia Dermato Funcional da Universidade Cidade de São Paulo (UNICID) - São Paulo (SP), Brasil.

${ }^{2}$ Fisioterapeuta; Mestre em Ciências pela Universidade Federal de São Paulo (Unifesp); Docente dos cursos de graduação em fisioterapia do Centro Universitário São Camilo e da Universidade Municipal de São Caetano do Sul (USCS) e do Curso de PósGraduação Lato Sensu em Fisioterapia Dermato Funcional da UNICID - Sâo Paulo (SP), Brasil.

${ }^{3}$ Fisioterapeuta; PhD em Ciências pela Unifesp; Coordenador do Curso de PósGraduação Lato Sensu em Fisioterapia Dermato Funcional; Docente do Programa de Pós-Graduação Stricto Sensu em Fisioterapia da Unicid; Professor colaborador do Programa de Pós-Graduaç̧ão Stricto Sensu em Cirurgia Plástica da UUNIFESP.

\section{ENDERECO PARA CORRESPONDÊNCIA:} Rogério Eduardo Tacani - Rua Sapucaia, 974, apto 83 - Mooca CEP 03170-050São Paulo (SP), Brasil - E-mail: rtacani@ uol.com.br

APRESENTACÃO maio 2010

ACEITO PARA PUBLICAC̄̃̃O set. 2010

FONTE DE FINANCIAMENTO: nenhuma

CONFLITO DE INTERESSE: nada a declarar

Trabalho aprovado pelo Comitê de Ética e Pesquisa da Unicid em 25 de junho de 2009, sob número PP 13428829.
RESUMO: Atualmente, a drenagem linfática manual (DLM) é um dos recursos de grande destaque no tratamento de edemas, linfedemas e condições inestéticas, porém, na prática clínica têm-se observado sua aplicação de forma iatrogênica. Os objetivos deste relato de caso foram apontar as sequelas da aplicação iatrogênica da DLM, em uma jovem caucasiana, e verificar os efeitos da intervenção fisioterapêutica no tratamento dessas. Voluntária de 27 anos, apresentava-se com uma equimose importante na região lateral da coxa esquerda $(28 \times 9,5 \mathrm{~cm})$, telangiectasias, microvaricosidades e petéquias em ambas as coxas e pernas, dor de forte intensidade e edema, após ter se submetido a um procedimento manual iatrogênico com finalidade estética há dois dias. As condutas fisioterapêuticas adotadas foram: Ultrassom $3 \mathrm{MHz}$, pulsado (2 ms on, $8 \mathrm{~ms}$ off), 0,4 W/cm² SATP na região da equimose e DLM (Método Leduc) durante 20 minutos, na coxa esquerda. Foram realizadas nove sessões até a alta no $16^{\circ}$ dia pós-lesão, observando-se apenas a redução gradativa da dor e da equimose. Verificou-se a efetividade da intervenção fisioterapêutica em apenas algumas das sequelas provocadas pela iatrogenia, sem desconsiderar que a remissão espontânea dos sinais e sintomas também pode ter contribuído para tal. Destaca-se a importância de conscientizar os fisioterapeutas que atuam com este procedimento para que o aplique de forma adequada, evitando tais iatrogenias.

Descritores: sistema linfático; massagem; doença iatrogênica; terapia por ultrassom; estética.

ABSTRACT: Recently, the manual lymphatic drainage (MLD) is an important resource in the treatment of edemas, lymphedemas, and other unaesthetic conditions. However, problems caused by the incorrect application of this therapy have been observed in the clinical practice. The aims of this case report were to describe sequelae resulting from the negligent application of MLD on a young patient, and to determine the effects of physical therapy on the treatment of sequelae. A 27-year-old Caucasian woman presented with a large ecchymosis $(28 \times 9.5 \mathrm{~cm})$ on the outer side of the left thigh, telangiectasias, micro varicose veins, and petechiae on the legs and thighs, intense pain, and edema, after undergoing an incorrect manual procedure for aesthetic purposes two days earlier. Physical therapy consisted of ultrasound application ( $3 \mathrm{MHz}$, pulsed mode, $20 \%$ duty cycle, SATP intensity of $0.4 \mathrm{~W} / \mathrm{cm}^{2}$ ) to the ecchymosis site and MLD (Leduc method) for 20 minutes on the left thigh. The patient underwent nine physical therapy sessions until discharge from the hospital 16 days after injury. The effectiveness of physiotherapy intervention was verified in only some of the consequences caused by iatrogenic, without disregarding that the spontaneous remission of signs symptoms can have contributed to such fact. The study emphasizes the importance of awareness among physical therapists who work with this procedure to apply it appropriately in order to avoid such conditions.

KeYwords: lymphatic system; massage; iatrogenic disease; ultrasonic therapy; esthetics. 


\section{INTRODUCÃO}

Diversas técnicas de massagem e terapias manuais têm sido usadas ao longo dos tempos para tratar condições inestéticas. Atualmente, devido ao maior interesse da população por tratamentos que melhorem os contornos corporais, as adiposidades localizadas e o fibro edema geloide, tem havido grande procura por diferentes recursos terapêuticos e massagens ditos como "redutores" ou "modeladores"1-4.

Por conta disso, observa-se uma crescente oferta destas técnicas em serviços e clínicas de estética, muitos destes gerenciados ou aplicados por profissionais não qualificados, os quais, aproveitando-se da popularidade das técnicas de drenagem linfática, têm seduzido um público cada vez maior, com falsas promessas de melhora imediata da condição estética e do bem-estar ${ }^{5}$.

Também têm surgido, de forma crescente, relatos de pacientes que frequentam estes serviços sobre características obscuras destas práticas em que são submetidos, em especial das técnicas de drenagem linfática, executadas de forma iatrogênica com manobras extremamente vigorosas, provocando dores intensas e equimoses, gerando um intenso sofrimento e complicações como microvarizes, piora do fibro edema geloide e deslocamento de trombos ${ }^{6}$.

Este panorama, que beira a margem do conhecimento científico, não apenas põe em risco a saúde da população ${ }^{7}$, como também pode contribuir para o descrédito e a desvalorização da Fisioterapia Dermato Funcional perante a sociedade leiga e científica.

Em função disto, os propósitos deste estudo foram apontar as sequelas da utilização iatrogênica da técnica de drenagem linfática manual em uma jovem caucasiana e verificar os efeitos da intervenção fisioterapêutica no tratamento destas.

\section{METODOLOGIA}

Descrição do caso

Voluntária do gênero feminino, 27 anos, caucasiana, queixa-se de dores e hematoma na região lateral da coxa esquerda. Relata que se submeteu a uma sessão de drenagem linfática manual (DLM), com finalidade estética há dois dias e cujas manobras, caracterizadas por deslizamentos e amassamentos "fortes" e "rápidos", foram executadas durante 75 minutos nas regiões do abdome e das coxas. Refere ter sentido dor intensa durante todo o procedimento, porém de modo insuportável na região lateral da coxa esquerda, solicitando a interrupção da técnica, porém a dor não cessou e, após algumas horas, notou o aparecimento de hematomas e inflamação na região.

Ao exame físico, observou-se uma equimose importante na região lateral da coxa esquerda, medindo aproximadamente $28 \times 9,5 \mathrm{~cm}$ (comprimento $\mathrm{x}$ largura), telangiectasias, microvaricosidades e petéquias esparsas em ambas as coxas e pernas, com índice de massa corporal IMC 23,75 kg/m², biotipo ginoide, pele fototipo II de Fitzpatrick e dor de forte intensidade $(7,5$ na escala visual analógica - EVA $)^{8}$ à palpação suave, tumefação tecidual local (edema ++/4; moderado), consistência do tecido subcutâneo levemente aumentada, temperatura discretamente elevada e sinal de Godet negativo ${ }^{9}$.

A pesquisa foi aprovada pelo Comitê de Ética e Pesquisa da Universidade Cidade de São Paulo (UNICID), n 13428829.

\section{Objetivos e condutas}

Frente a esse quadro, os objetivos terapêuticos foram reduzir o quadro inflamatório, a dor, o edema e as equimoses, por meio das seguintes condutas fisioterapêuticas:

- Ultrassom $3 \mathrm{MHz}$, pulsado com ciclo de trabalho de 20\% (2 ms on, 8 ms off), densidade de potência de $0,4 \mathrm{~W} / \mathrm{cm}^{2}$ (Spatial Average Temporal Peak - SATP), frequencia de repetição de pulso de $100 \mathrm{~Hz}$, área de radiação efetiva (Effective Radiation Area - ERA) de $5 \mathrm{~cm}^{2}$, aplicado de forma circular, uniforme, ininterrupta e lenta (aproximadamente $4 \mathrm{~cm} /$ segundos) com gel acoplante (polímero carboxivinílico). A área selecionada foi a região central da equimose, dividida em dois quadrantes, Q1 (inferior) de $57 \mathrm{~cm}^{2}$ e Q2 (superior) de $47 \mathrm{~cm}^{2}$ de área aproximada, durante 11 e 9 minutos, respectivamente. $\mathrm{O}$ tempo foi calculado de acordo com a fórmula: tempo = área/ERA $x 1,5^{10}$. O ultrassom utilizado foi o Sonacel Expert ${ }^{\circledR}$ de $3 \mathrm{MHz}$, calibrado com dosímetro de precisão (Ultrasonic Power Meter, modelo UPM-DT10), ambos da Bioset Indústria de Tecnologia Eletrônica Ltda, Rio Claro, SP, Brasil.

- DLM, técnica Leduc e Leduc ${ }^{11}$ durante 20 minutos na coxa esquerda, com pressão suave (de aproximadamente até $40 \mathrm{mmHg}$, aplicada por profissional experiente e previamente treinado) e velocidade lenta (aproximadamente uma manobra a cada cinco segundos), executandose dez manobras nos nódulos linfáticos supraclaviculares e nos inguinais esquerdos, manobras em reabsorção (Résorption) ${ }^{11}$ na coxa medial, anterior e lateral até a extremidade distal da equimose, repetindo-se dez vezes a cada 5 $\mathrm{cm}$, retornando com manobras em chamada ( $D^{\prime}$ appel $)^{11}$ até a região proximal da coxa, com a mesma distância e repetição e finalizando com 10 manobras nos nódulos linfáticos inguinais esquerdos.

As orientações gerais foram: evitar novos traumas e temperaturas muito elevadas no local.

\section{Procedimentos de avaliação}

A evolução da dor foi quantificada por meio da $\mathrm{EVA}^{8}$, do edema, por meio da inspeção e palpação ${ }^{9}$, e das equimoses por registro fotográfico com câmera digital de 7.2 megapixels, modelo DSCW110 Sony ${ }^{\circledR}$, posicionada perpendicularmente a $30 \mathrm{~cm}$ da coxa esquerda ${ }^{12}$, para graduação do espectro equimótico de Legrand du Saulle ${ }^{13}$, ambos coletados antes dos procedimentos terapêuticos a cada atendimento.

\section{RESULTADOS}

Foram realizadas nove sessões de tratamento até a alta da voluntária, num período total de 14 dias, executadas no $2^{\circ}, 3^{\circ}, 4^{\circ}, 5^{\circ}, 6^{\circ}, 10^{\circ}, 11^{\circ}, 12^{\circ}$ e $16^{\circ}$ dias após a lesão, seguindo-se as mesmas condutas. 
Observou-se que a dor da voluntária inicialmente de forte intensidade $(7,5)$ passou a ser moderada $(5,0)$ após a $1^{\text {a }}$ sessão, de fraca intensidade da $5^{a}$ $(3,0)$ à $10^{\mathrm{a}}(1,0)$ sessão e cessou na $11^{\mathrm{a}}$, mantendo-se ausente até a alta, conforme apontado na Figura 1.

O edema diminuiu após a primeira sessão, passando de moderado $(++/ 4)$ a leve $(+/ 4)$ e regrediu completamente na quinta sessão. $\mathrm{O}$ aspecto da equimose teve evolução gradativa, com discreta diminuição da tonalidade vermelho violácea do segundo ao sexto dia póslesão, porém com importante redução da tonalidade vermelho violácea para verde-amarelada do $6^{\circ}$ ao $11^{\circ}$ dia e clareamento quase total do $11^{\circ}$ ao $16^{\circ}$ dia, permanecendo apenas discretas equimoses verde-amareladas no bordo lateral do quadrante inferior. No $16^{\circ} \mathrm{dia}$, verificou-se a presença de telangiectasias e microvaricosidades na área da lesão, sendo que a voluntária declarou não apresentar tais lesões antes do procedimento iatrogênico inicial. As Figuras 2, 3 e 4 ilustram a evolução do edema e das equimoses.

\section{DISCUSSÃO}

Apesar da existência de versões adaptadas e evoluídas relacionadas à DLM, que são aprimoradas por diferentes escolas científicas ao longo da história, conforme achados e pesquisas experimentais de anatomia, fisiologia e fisiopatologia do sistema linfático, as manobras devem ser sempre leves, superficiais, lentas, pausadas e repetitivas, drenando apenas o líquido intersticial dos tecidos mais superficiais do corpo e a rede de plexos linfovenosos subpapilares, intradérmicos e hipodérmicos, localizados entre as camadas da pele e tela subcutânea. Devido a isto, a pressão exercida sobre a pele do paciente deve ser de até 30 a $40 \mathrm{mmHg}^{14-16}$.

O que ocorre nas diversas situações de seu uso, em indivíduos saudáveis ou com doenças e lesões, são adaptações de alguns componentes da técnica, conforme o quadro clínico individual vigente, porém sem descaracterizá-la ${ }^{16-21}$. Os edemas, linfedemas e outros distúrbios correlacionados podem apresentar um caráter inestético, e a melhora clínica

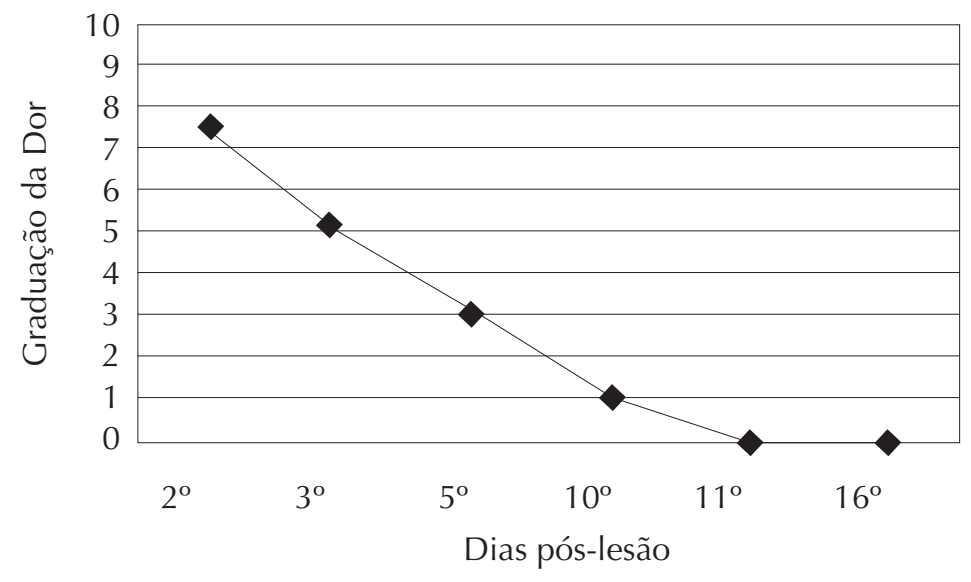

Figura 1. Evolução do quadro álgico pela escala visual analógica

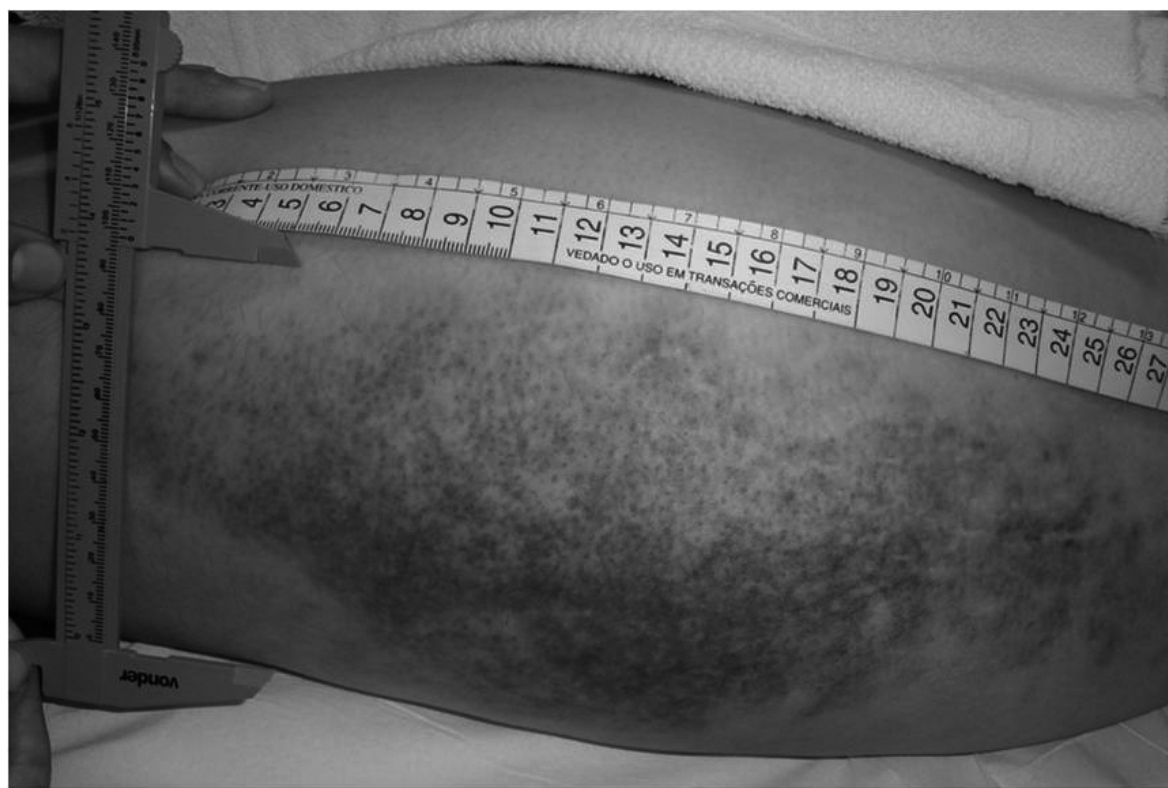

\section{$2^{\circ}$ dia de lesão; Pré-tratamento.}

Figura 2. Região submetida à aplicação iatrogênica da drenagem linfática manual, apresentando uma equimose de $28 \times 9,5 \mathrm{~cm}$ no segundo dia de lesão antes de iniciar o tratamento fisioterapêutico

destas condições patológicas normaliza a função dos órgãos e sistemas orgânicos, influenciando, consequentemente, a aparência corporal dos indivíduos e, despertando grande interesse na população pela DLM.

Aproveitando-se disto, muitos serviços de estética e embelezamento nos últimos anos passaram a oferecer estes tratamentos ao público, sendo notificados, no estado de São Paulo (até outubro de 2007) pelo Conselho Regional de Fisioterapia e
Terapia Ocupacional da Terceira Região (CREFITO 3), cerca de 1.300 estabelecimentos, que faziam o uso indevido do termo drenagem linfática e ofereciam a técnica sem profissionais habilitados ${ }^{5}$.

A voluntária, quando questionada se o profissional que a atendeu era habilitado, respondeu negativamente, o que denota a necessidade de fiscalizações constantes dos Conselhos Regionais de Fisioterapia e a execução de campanhas educativas para a população. 
Godoy, Belczack e Godoy²2 alertam que várias técnicas de massagem são utilizadas de maneira inadequada e denominadas falsamente de DLM, causando prejuízos aos pacientes, como Casley-Smith et al. ${ }^{14}$ relatam que uma DLM mal aplicada é um dos piores

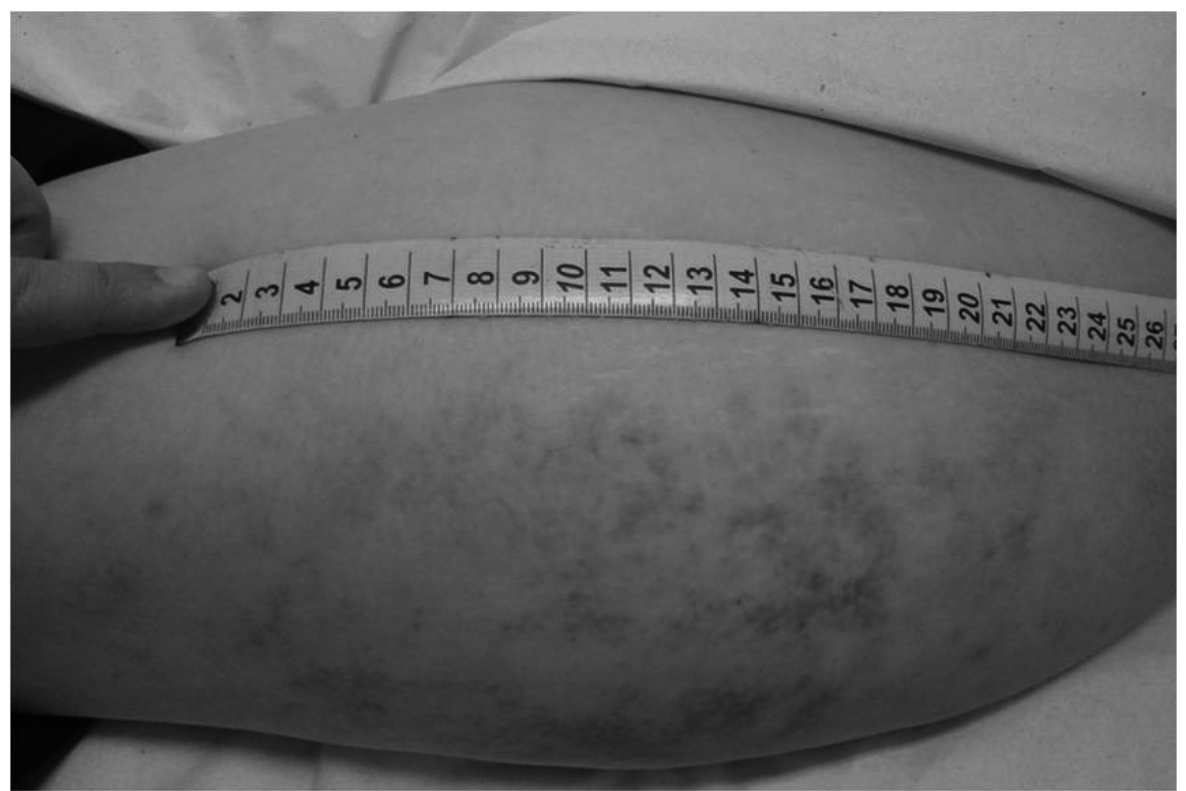

\section{$11^{\circ}$ dia de lesão; $7^{\mathrm{a}}$ sessão de tratamento.}

Figura 3. Evolução da equimose no $11^{\circ}$ dia pós-lesão, com importante redução da tonalidade vermelho violácea - sétima sessão de atendimento

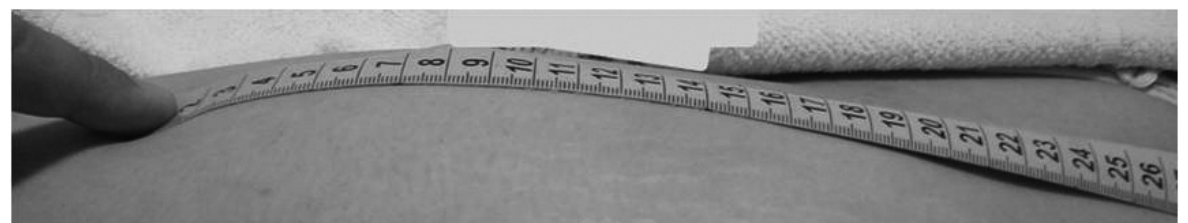

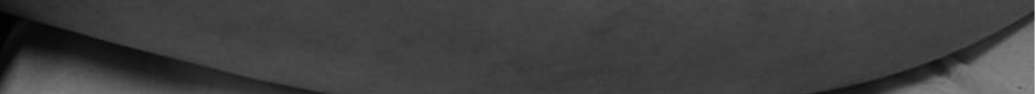

\section{$16^{\circ}$ dia de lesão; Pós-tratamento ( $9^{\mathrm{a}}$ sessão).}

Figura 4. Evolução da equimose no $16^{\circ}$ dia pós-lesão, notar a presença de telangiectasias e microvaricosidades na área da lesão - nona sessão de atendimento
Outras técnicas de massagens hiperemiantes e de descolamento subcutâneo, utilizando manobras com maior pressão que a DLM, são indicadas como coadjuvantes para o tratamento de algumas disfunções estéticas, porém, devem respeitar a integridade dos tecidos manipulados e a sensibilidade dolorosa dos pacientes, especialmente daqueles que apresentam fragilidade capilar $^{23}$, como no caso da voluntária em questão.

A propósito, segundo Heckel apud Boigey ${ }^{24}$, "[...] a idéia muito difundida, há uns 20 anos, de amassar os nódulos e as placas de celulite através de beliscão energético indo até a equimose, é a prova de um incurável obscurantismo", mostra que há quase 90 anos já se descrevia que as técnicas de massagem dos tecidos superficiais com fibro edema geloide (celulite) e adiposidades localizadas devem ser realizadas de forma leve, superficial, branda e agradável, respeitando sua integridade, para não produzir equimoses e tampouco dor excessiva, uma vez que a ruptura das fibras elásticas e a formação de processos inflamatórios pioram ainda mais o estado dos tecidos comprometidos ${ }^{23,24}$.

Observou-se, neste caso, que a aplicação iatrogênica da DLM provocou um intenso processo inflamatório associado à equimose na região lateral da coxa esquerda e que, mesmo após a intervenção fisioterapêutica, persistiram lesões como telangiectasias e microvaricosidades, conforme relato da voluntária, corroborando com a inadequação deste tipo de recurso manual e demonstrando que pode provocar danos sérios à funcionalidade tecidual e à própria condição da estética corporal.

Além disso, a mesma também foi submetida à massagem na região abdominal e, embora nã o tenha apresentado equimoses subcutâneas, foi exposta aos riscos apontados por Trotter ${ }^{25}$, que descreve outro caso de iatrogenia por massagem vigorosa para finalidades estéticas, de maior gravidade. O caso de uma mulher de 39 anos, submetida à massagem corporal profunda na região abdominal, incluindo o quadrante superior direito, que após 24 horas apresentou desconforto abdominal 
vagal, náuseas e dores no ombro direito, sendo hospitalizada com alterações graves do hematócrito, células brancas, albumina, enzimas hepáticas, tempo de protrombina e um grande hematoma de 14 x $18 \mathrm{~cm}$ no lobo hepático direito detectado por tomografia. Em seguida, foi tratada com transfusão de sangue e nos seis meses seguintes a mesma emagreceu 10,4 kg em função das náuseas e estado febril, mesmo em regime de internação.

Outras sequelas, como necrose de tecido adiposo ${ }^{26}$, perda auditiva neurossensorial após massagem no músculo trapézio, lesão da artéria vertebral ${ }^{27}$, deslocamento ureteral, síndrome interóssea anterior, pseudo-aneurisma arterial poplíteo ${ }^{28}$, também vêm sendo referidas, mesmo que sejam de forma isolada e em número reduzido.

Entretanto, estes casos não podem ser negligenciados ${ }^{26,27}$, merecendo destaque e atenção da população, autoridades e das associações de fisioterapia dermato funcional, que também devem se sensibilizar e contribuir para a eliminação deste panorama, pois, segundo a Resolução CREFITO-3 n ${ }^{\circ} 27$, de 29 de setembro de 2007, a técnica de drenagem linfática deve ser exercida por fisioterapeutas para a promoção da saúde e proteção da integridade física da pessoa humana ${ }^{7}$.

A redução da dor verificada pela EVA, demonstrada na Figura 1, pode ter ocorrido pela resolução do edema ${ }^{29}$ e do quadro inflamatório incentivado pela ação pró-inflamatória do ultrassom, que estimula a liberação de mediadores químicos como a serotonina, por aumentar a permeabilidade da membrana das plaquetas, e a histamina pela degranulação de mastócitos, bem como fatores de crescimento de fibroblastos pelos macrófagos, dentre outros eventos que aceleram a fase inflamatória, evoluindo mais precocemente para a fase proliferativa do reparo tecidual ${ }^{30-33}$.

Soma-se aos efeitos do ultrassom, a ação antiedematosa da DLM devido à descompressão das terminações nervosas, além do efeito vagotônico com ação sedativa e analgésica, desencadeada pelos estímulos parassimpáticos, provenientes das manobras da técnica ${ }^{20,23}$. Neste caso, foram utilizadas inicialmente as manobras de reabsorção (Résorption) $)^{11}$, que aceleram consideravelmente a reabsorção do edema e de macromoléculas pelos linfáticos iniciais, aumentando a formação de linfa. Em seguida, foram realizadas as manobras de chamada ( $D^{\prime}$ appel) ${ }^{11}$ que exercem efeito de aspiração linfática, aumentando o transporte da linfa recémcaptada ao longo dos vasos linfáticos, conforme preconizado por Leduc, Bourgeois, Leduc ${ }^{34,35}$.

Quanto à evolução da equimose da coxa esquerda, observou-se sua resolução com sete sessões de atendimento, provavelmente porque o ultrassom acelera a reabsorção de edemas e hematomas e atua, de forma fibrinolítica e trombolítica ${ }^{36,37}$. Berná-Serna et al. ${ }^{36}$ relatam um caso parecido com o deste estudo, porém, a voluntária de 62 anos apresentava um hematoma na bainha do reto abdominal de $12 \times 8 \mathrm{~cm}$ e foi tratada com ultrassom $1 \mathrm{MHz}, 1,5$ a 2,0 $\mathrm{W} / \mathrm{cm}^{2}, 8$ a 12 minutos, pulsado com ciclo de trabalho de $20 \%$, cinco vezes por semana, reduzindo de 12 para $5 \mathrm{~cm}$ em apenas um mês, quando comparado ao tratamento convencional de três meses ou mais.

Selecionou-se a frequência do transdutor de $3 \mathrm{MHz}$, pois a energia ultrasônica é absorvida entre 1 ou $2 \mathrm{~cm}$ de profundidade $^{38}$, o que é suficiente para atingir a lesão da coxa esquerda da voluntária, diferentemente de Berná-Serna et al. ${ }^{36}$, que utilizaram $1 \mathrm{MHz}$, pois o hematoma estava em uma região mais profunda. Ademais, a $3 \mathrm{MHz}$ a energia ultrasônica é absorvida três vezes mais rápido que a $1 \mathrm{MHz}^{38}$, podendo-se em casos subagudos considerar o tempo em 1,5 a menos do resultado obtido ${ }^{10}$. Assim, viabiliza-se a prática clínica com relação ao tempo de aplicação, especialmente neste caso, cuja área da equimose era muito extensa. O ciclo de trabalho de $20 \%$ foi estipulado devido ao tratamento ter iniciado ainda na fase aguda, na qual não se deseja a produção e o acúmulo de calor, promovidos pela modalidade contínua ${ }^{38}$ e corrobora com o utilizado por Berná-Serna et al. ${ }^{36}$.

A persistência das discretas equimoses verde-amareladas no bordo lateral do quadrante inferior, no $16^{\circ} \mathrm{dia}$, pode ter ocorrido por esta área ser a mais distal dos nódulos inguinais esquerdos e por apresentar menor número de coletores linfáticos, dificultando a reabsorção, além de ser uma região com mais adiposidade nas mulheres, o que facilita o acúmulo de líquido intersticial, edemas ou lipedemas. De acordo com o espectro equimótico de Legrand du Saulle ${ }^{13}$, após 16 dias, a pele já tende a obter a cor natural novamente, fato que culmina com a remissão espontânea, a qual pode ter colaborado com os resultados observados, independentemente do tratamento fisioterapêutico.

\section{CONCLUSÃO}

A aplicação iatrogênica da técnica de DLM, no caso estudado, desencadeou intenso processo inflamatório associado à equimose na região lateral da coxa esquerda, telangiectasias, microvaricosidades, petéquias, dor de forte intensidade e edema, demonstrando a inadequação deste tipo de recurso manual, o que pode provocar danos sérios à funcionalidade tecidual e à própria condição estética corporal da voluntária, além da possibilidade de prejuízos à saúde.

O ultrassom associado à DLM favoreceu a reabsorção do edema e da equimose, estimulou a resolução do processo inflamatório e aliviou a dor, verificando-se a efetividade da intervenção fisioterapêutica em apenas algumas das sequelas provocadas pela iatrogenia, sem desconsiderar que a remissão espontânea dos sinais e sintomas também pode ter contribuído para tal fato.

A DLM, independentemente da finalidade ou estilo de técnica, respeita a anatomia e fisiologia do sistema linfático e a integridade dos tecidos superficiais. Para tanto, deve ser executada de forma suave, lenta e rítmica, sem causar, em hipótese alguma, danos ou lesões aos tecidos e dor ao paciente. Por isso, cabe ressaltar a importância de conscientizar os fisioterapeutas que atuam com este procedimento para que o aplique de forma adequada, evitando tais iatrogenias, bem como a necessidade do desenvolvimento de ensaios clínicos controlados para avaliar a efetividade das técnicas fisioterapêuticas realizadas. 


\section{REFERÊNCIAS}

1. Pedini G, Zaietta P. Su alcuni aspetti della attivazione della lipolisi tessutale da parte di fattori meccanici. Minerva Med. 1975;66:318-23.

2. Lucassen GW, van der Sluys WLN, Vanherk JJGW, Nuijs AM, Wierenga PE, Barel AO, et al. The effectiveness of massage treatment on cellulite as monitored by ultrasound imaging. Skin Res Technol. 1997;3:154-60.

3. Collis N, Elliot L-A, Sharpe C, Sharpe DT. Cellulite treatment: a myth or reality: A prospective randomized, controlled trial of two therapies, endermologie and aminophylline cream. Plast Reconstr Surg. 1999;104:1110-4.

4. Tunay VB, Akbayrak T, Bakar Y, Kayihan H, Ergun N. Effects of mechanical massage, manual lymphatic drainage and connective tissue manipulation techniques on fat mass in women with cellulite. J Eur Acad Dermatol Venereol 2010;24:138-42.

5. Conselho Regional de Fisioterapia e Terapia Ocupacional da $3^{\text {a }}$ Região. Massoterapia e Drenagem Linfática: denuncie a prática ilegal dessas técnicas. [serial online] 2008 Jul [Citado em 3 Jul 2008]. Disponível em http://www.crefito.com.br/admin/monta_temp11. asp?cd_templa $=368$.

6. Conselho Regional de Fisioterapia e Terapia Ocupacional da $3^{a}$ Região. Ministério Público de Ribeirão Preto investiga drenagem. [serial online] 2008 Jul [Citado em 28 Jul 2008]. Disponível em http://www.crefito.com.br/ admin/monta_temp11.asp?cd_templa=318.

7. Brasil. Ministério do Trabalho. Resolução CREFITO-3 $\mathrm{n}^{\circ}$. 127, de 29 de setembro de 2007. Dispõe de modo complementar à Resolução COFFITO nº. 08/1978 quanto à aplicação da drenagem linfática. [Citado em 29 Set 2007]. Disponível em http://www.crefito.com.br/.

8. Breivik EK, Björnsson GA, Skovlund E. A Comparison of Pain Rating Scales by Sampling From Clinical Trial Data. Clin J Pain. 2000;16:22-8.

9. Diskin CJ, Stokes TJ, Dansby LM, Carter TB, Radcliff L, Thomas SG. Towards an understanding of oedema. BMJ. 1999;318:1610-3.

10. Hecox B, Mehreteab TA, Wisberg J. Physical agents: a comprehensive text for physical therapists. Connecticut: Appleton \& Lange, 1994.

11. Leduc A, Leduc O. Le drainage lymphatique: théorie et

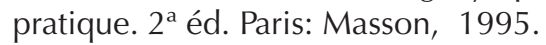

12. Hochman B, Nahas FX, Ferreira LM. Fotografia aplicada na pesquisa clínico-cirúrgica. Acta Cir Bras. 2005;20 Suppl.2:19-25.

13. Alcântara HR. Perícia Médica Judicial. 2ª ed. Rio de Janeiro: Guanabara Koogan; 2006.
14. Casley-Smith JR, Boris M, Weindorf S, Lasinski B. Treatment for lymphedema of the arm - The Casley-Smith method. Cancer. 1998;83:2843-58.

15. Kasseroller RG. The Vodder school: The Vodder method. Cancer 1998;83:2840-2.

16. Badger C, Seers K, Preston N, Mortimer P. Physical therapies for reducing and controlling lymphoedema of the limbs (Protocol for a Cochrane Review). Cochrane Library 2002; issue 1. Oxford: Update Software.

17. Moseley AL, Caratiz CJ, Piller NB. A systematic review of common conservative therapies for arm lymphoedema secondary to breast cancer treatment. Ann Oncol. 2007;18:639-46.

18. Rubin A, Hoefflin SM, Rubin M. Treatment of postoperative bruising and edema with external ultrasound and manual lymphatic drainage. Plast Reconstr Surg. 2002;109:1469-71.

19. Soares LMA, Soares SMB, Soares AKA. Estudo comparativo da eficácia da drenagem linfática manual e mecânica no pós-operatório de dermolipectomia. Rev Bras Prom Saúde. 2005;18:199-204.

20. Tacani RE, Gimenes RO, Alegrance FC, Assumpção JD. Investigação do encaminhamento médico a tratamentos fisioterapêuticos de pacientes submetidos à lipoaspiração. Mundo Saúde. 2005;29:192-8.

21. Arieiro EG, Machado KS, Lima VP, Tacani RE, Diz AM. A eficácia da drenagem linfática manual no pós-operatório de câncer de cabeça e pescoço. Rev Bras Cir Cabeça Pes 2007;36:43-6.

22. Godoy JMP, Belczack CEQ, Godoy MFG. Reabilitação linfovenosa. Rio de Janeiro: Dilivros, 2005.

23. Tacani RE, Cervera L. Técnicas manuais. In: De Maio M, editor. Tratado de Medicina Estética. São Paulo: Roca; 2004:1881-918.

24. Boigey M. Manual de Massagem. São Paulo: Andrei, 1986.

25. Trotter JF. Hepatic hematoma after deep tissue massage. N Engl J Med. 1999;341:2019-20.

26. Hanif Z, Ahmad M. Subcutaneous fat necrosis presenting as large mass. Eur J Emerg Med. 2006;13:106-7.

27. Grant KE. Massage safety: injuries reported in Medline relating to the practice of therapeutic massage: 1965 2003. J Bodyw Mov Ther. 2003;7:207-12.

28. Ernst E. The safety of massage therapy. Rheumatology. 2003;42:1101-6.

29. Draper DO, Castel JC, Castel D. Rate of temperature increase in human muscle during $1 \mathrm{MHz}$ and $3 \mathrm{MHz}$ 


\section{Referências (cont.)}

continuous ultrasound. J Orthop Sports Phys Ther. 1995;22(4):142-50.

30. Dyson M. Mechanisms involved in therapeutic ultrasound. Physiotherapy. 1987;73:116-20.

31. Young SR, Dyson M. Macrophage responsiveness to therapeutic ultrasound. Ultrasound Med Biol. 1990;16:809-16.

32. Young SR, Dyson M. Effect of therapeutic ultrasound on the healing of full-thickness excised skin lesions. Ultrasonics. 1990;28(3):175-80.

33. Paliwal S, Mitragotri S. Therapeutic opportunities in biological responses of ultrasound. Ultrasonics. 2008;48:271-8.

34. Leduc O, Bourgeois P, Leduc A. Approche expérimentale de l'influence du drainage lymphatique manuel par lymphographie isotopique. Ann Kinésithér 1988; 15:153-8.

35. Leduc O, Bourgeois P, Leduc A. Manual Lymphatic Drainage scintigraphic demonstration of its efficacy on colloidal protein reabsorption. Progress in Lymphology. 1988;11:551-4

36. 'Berná-Serna JD, Sánchez-Garre J, Madrigal M, Zuazu I, Berná-Mestre JD. Ultrasound therapy in rectus sheath hematoma. Phys Ther. 2005;85:352-7.

37. Kornowski R, Meltzer RS, Chernine A, Vered Z, Battler A. Does external ultrasound accelerate thrombolysis? Results from a rabbit model. Circulation. 1994;89:339-44.

38. Draper DO, Ricard MD. Rate of temperature decay in human muscle following $3 \mathrm{MHz}$ ultrasound: The stretching window revealed. J Athl Train. 1995;30:304-7. 